\title{
Effect of Mind-Body Approaches on Menopausal Symptoms among Women: A Community-Based Pilot Study
}

\author{
Timi Thomas ${ }^{1}$ Neetha Kamath ${ }^{2}$ Ajay Kumar ${ }^{3}$ Fatima D'Silva ${ }^{4}$ \\ ${ }^{1}$ Department of Obstetrics and Gynaecological Nursing, Nitte Usha \\ Institute of Nursing Sciences, Nitte (Deemed to be) University, \\ Mangalore, Karnataka, India \\ ${ }^{2}$ Department of Community Health Nursing, Nitte Usha Institute \\ of Nursing Sciences, Nitte (Deemed to be) University, Mangalore, \\ Karnataka, India \\ ${ }^{3}$ Behavioural Medicine Unit and Adult Psychiatry Unit, NIMHANS, \\ Bangalore, Karnataka, India \\ ${ }^{4}$ Department of Medical-Surgical Nursing, Nitte Usha Institute of \\ Nursing Sciences, Nitte (Deemed to be) University, Mangalore, \\ Karnataka, India
}

\begin{abstract}
Address for correspondence Neetha Kamath, PhD, Department of Community Health Nursing, Nitte Usha Institute of Nursing Sciences, Nitte (Deemed to be) University, Mangalore 575018, Karnataka, India (e-mail: neetha.jayavanth@gmil.com).
\end{abstract}

J Health Allied Sci ${ }^{\mathrm{Nu}: 2021 ; 11: 61-65}$

\begin{abstract}
Keywords

- mind-body approach

- Mindfulness

meditation

- menopausal symptoms

- menopausal women
\end{abstract}

Introduction The process of women aging is characterized by a gradual transition from reproductive to nonreproductive life period. These changes in aging during menopause are accompanied by a wide range of physiological as well as psychological disturbing symptoms. The traditional therapeutic approach toward menopause management is hormone replacement therapy, which increases the risk of breast cancer, stroke, and coronary heart disease. However, the efficacy of complementary and alternative therapies like mind-body approach to troublesome hot flashes, anxiety, and depression is widely proven and it is used by many women. This study aimed to evaluate the effectiveness of the mind-body approach on menopausal symptoms among women.

Methods A quantitative research approach with quasi-experimental pretestpost-test design was adopted in the study. A total of 20 samples between the ages of 48 to 55 years with menopausal symptoms were the participants. The intervention of mind body approach (Mindfulness meditation) was performed for 8 weeks, which includes 35 minutes of session per day.

Results The majority ( $80 \%$ ) of the participants were in the age group of 51 to 53 years and $50 \%$ of the participants attained menopause between 49 and 50 years. The results showed that the mean post-test menopausal symptom score was $15.60 \pm 2.633$ which was lower than the mean pretest score $28.420 \pm 1.476$ and the calculated $t$-value, $t=10.157$ is greater than the table value $(p<0.001)$ at 0.001 level of significance. Hence, it is evident that the practice of mind-body approach was effective in reducing the intensity of menopausal symptoms among women. published online January 21, 2021
DOI https://doi.org/ $10.1055 / \mathrm{s}-0040-1722423$ ISSN 2582-4287.
(C) 2021. Nitte (Deemed to be University).

This is an open access article published by Thieme under the terms of the Creative Commons Attribution-NonDerivative-NonCommercial-License, permitting copying and reproduction so long as the original work is given appropriate credit. Contents may not be used for commercial purposes, or adapted, remixed, transformed or built upon. (https://creativecommons.org/licenses/by-nc-nd/4.0/).

Thieme Medical and Scientific Publishers Pvt. Ltd. A-12, 2nd Floor, Sector 2, Noida-201301 UP, India 
Conclusion The study concludes that the practice of mind-body approach helped in reducing the menopausal symptoms on the domain of psychological and Somato vegetative scale like anxiety, depression, reduction in the intensity of hot flushes, and improved the sleeping pattern. Overall, the practice of Mindfulness meditation helped the participants to have a better quality of life. The study was found to be cost-effective and feasible to be made as a routine intervention for women to manage menopausal symptoms.

\section{Introduction}

The number of postmenopausal women has increased in the world because of the enhanced lifestyle practices. With the aging of the worldwide population in the coming decades, it is estimated that 1.2 billion women worldwide will be menopausal or postmenopausal by the year $2030^{1}$ and the common symptoms of menopause include vasomotor symptoms (hot flushes [HFs] and/or night sweats), sleep disturbances, and vaginal dryness. ${ }^{2}$ According to the Indian National Family Health Survey performed between 2005 and 2006 , it was reported that nearly $18 \%$ of currently married women are in the age group of 30 to 49 years and reached menopause. ${ }^{3}$ The prevalence of vasomotor symptoms alone is estimated at approximately 40 to 50 million women in the United States. ${ }^{4}$ As per the statistics of the United States in $2000,27 \%$ of the population consisted of women 45 years and older, whereas now it is estimated to be increased to $38 \%{ }^{5}$ It is estimated that in the world wide nearly $85 \%$ of postmenopausal women have experienced menopause-related symptoms in their lifetime ${ }^{6}$ which makes them have impaired quality of life. In 2030, nearly half of females will become postmenopausal and by 2060, the proportion of postmenopausal women will be increased up to $59.8 \%{ }^{7}$ It has been estimated that the average age for the onset of menopause among Indian women is 47.5 years with an average life expectancy of 71 years. ${ }^{8}$ Whereas, the median age at natural menopause among Caucasian women in Western society is 50 years. $^{9}$

Considering the limitation of hormone replacement therapy (HRT), the health professionals are searching for different ways to manage menopause to minimize discomfort and inconvenience, to improve the quality of life among peri and postmenopausal women. ${ }^{10}$ The present study's need is to explore new options for the management of menopausal symptoms in the form of nonhormonal drug therapy and nonpharmacological measures. The current recommendations are diet, regular exercise, change in lifestyle, yoga, therapeutic massage, and other stress-reducing measures. Many treatments are available to date for the management of menopause symptoms. Among all the available treatments, dietary supplements, mind-body techniques, and yoga could play an effective role in the management of menopausal symptoms due to less cost, time, and effort. ${ }^{11}$ Moreover, these therapies have no side effects and from the literature, it is found to be statistically significant. Hence, this study aimed to assess the effectiveness of the mind-body approach to minimize menopausal symptoms among women.

\section{Methods}

\section{Study Design and Participants}

A quantitative research approach and quasi-experimental pretest-post-test design was adopted to find the effect of mind-body approaches on menopausal symptoms among women of Kotekar Primary Health Centre, Mangalore. A total of 20 samples between the ages of 48 to 55 years with menopausal symptoms who fulfilled the inclusion criteria were assigned into the study group. Exclusion criteria for study participants were: participants who were regularly practicing meditation and yoga, participants with surgical menopause, receiving any kind of HRT or anticoagulant therapy, participants with anxiety or mood disorders, depression, schizophrenia, diabetes, hypertension, and cardiac diseases.

\section{Data Collection Instruments}

The data were collected using the socio-demographic proforma, self-reported menopausal symptom checklist, and standardized menopausal rating scale (MRS). The sociographic proforma consists of six items such as the age of the women, age at which menopause is attained, educational status, nature of work of women, previous knowledge regarding menopause, and any history of gastrointestinal disorders. The self-reported menopausal checklist was used to allot the samples into the study group. The checklist consists of 29 menopausal symptoms, which were classified and scored as mild, moderate, and severe menopausal symptoms. Under the guidance of a gynecologist, it was finalized that menopausal women with moderate and severe symptoms with an average of 6 and above have been taken for the study. The level of menopausal symptoms was measured using the standardized MRS. The domains included in the MRS scale were psychological scale, Somato vegetative scale, and urogenital scale. The severity of menopausal symptoms is categorized as follows: 0 to 14 (mild), 15 to 29 (moderate), and 30 to 44 (severe). The standardized tools of the English version were translated to Kannada with the help of language experts and retranslation was done to ensure language validity. The internal consistency of the scales was calculated using the Karl Pearson correlation coefficient and Cronbach's $\alpha$ : MRS ( $r=0.889)$. 


\section{Ethical Considerations}

The Institutional Ethics Committee of Nitte Usha Institute of Nursing Sciences (Ref: NUINS/CON/ NU/IEC/2016-17) and the Central Ethics Committee of Nitte University, Deralakatte issued an ethical clearance certificate (Ref: NU/CEC/20182018/0143). The present study fits into the principles defined in the Declaration of Helsinki ${ }^{12}$ (World Medical Association, 2013). The study protocol was registered in CTRI and the number is CTRI/2017/07/009071.

\section{Data Collection Procedure}

Before the data collection, an informed written consent and participant information sheet was administered to the participants to assure confidentiality of data among the participants, followed which, the participants' menopausal symptoms were assessed using a self-reported menopausal symptom checklist. Once the participants were allotted to the study group their demographic proforma and preinterventional menopausal symptoms were assessed on the first day of selection. The participants in the study group received eight techniques of Mindfulness meditation (mind-body approach) i.e., counting 10 breaths and brief breathing meditation, Raisin meditation, Sitting meditation, Hatha yoga, Body scan meditation, Loving-kindness meditation, Mindful walking meditation, and Silent meditation. The mind-body approaches comprise an 8 weeks program which includes 35 minutes of session per day with the help of audio clippings. The sessions were led by the investigator. The investigator had training on Mindfulness meditation and yoga. For Mindfulness meditation, the investigator had been to Behavioral Medicine Unit, NIMHANS, Bangalore from August 01, 2019 to August 14, 2019, and for yoga at Pranava yoga and Naturopathy center, Mangalore. After 8 weeks, the post-test was taken, and the data analysis was done using SPSS 20.

\section{Results}

\section{Baseline Information}

The majority (80\%) of the participants were in the age group of 51 to 53 years and $50 \%$ of the participants attained menopause between 49 and 50 years. Regarding education, the majority of women had a high school education (60\%). About the nature of the work of women, all the menopausal women were semiskilled workers and their earnings are up to Rs. 6,662/-. All the menopausal women were married, and the majority (80\%) are from a nuclear family. The majority (70\%) of the menopausal women did not have previous information regarding menopause and its management and among the women who received the information are from family members (20\%) and health professionals (10\%) and none of the participants were reported with any kind of gastrointestinal disorders among the group (-Table $\mathbf{1}$ ).

The data in the - Table 2 shows that, the majority (70\%) of the participants in the study group had a moderate level of menopausal symptoms and $30 \%$ of the participants had severe level of menopausal symptoms with a mean of 28.2 \pm 1.476 . Whereas in the post-test, $80 \%$ of the participants
Table 1 Frequency ( $f$ ) and percentage (\%) distribution of demographic variables of participants

\begin{tabular}{|c|c|c|}
\hline \multicolumn{3}{|l|}{$n=20$} \\
\hline Demographic variables & $\begin{array}{l}\text { Frequency } \\
\text { (f) }\end{array}$ & $\begin{array}{l}\text { Percentage } \\
\text { (\%) }\end{array}$ \\
\hline \multicolumn{3}{|l|}{ Age in years } \\
\hline $48-50$ & 2 & 10 \\
\hline $51-53$ & 16 & 80 \\
\hline $54-55$ & 2 & 10 \\
\hline \multicolumn{3}{|l|}{ Age at menopause (years) } \\
\hline $45-46$ & 4 & 20 \\
\hline $47-48$ & 6 & 30 \\
\hline $49-50$ & 10 & 50 \\
\hline \multicolumn{3}{|l|}{ Educational status of woman } \\
\hline Primary education & 1 & 5 \\
\hline High school education & 15 & 75 \\
\hline Higher secondary education & 4 & 20 \\
\hline \multicolumn{3}{|l|}{ Nature of work of women } \\
\hline Semi-skilled worker & 20 & 100 \\
\hline \multicolumn{3}{|l|}{$\begin{array}{l}\text { Income status of women/month } \\
\text { (in INR) }\end{array}$} \\
\hline $2,425-6,662$ & 20 & 100 \\
\hline \multicolumn{3}{|l|}{ Type of family } \\
\hline Nuclear family & 18 & 90 \\
\hline Joint family & 2 & 10 \\
\hline \multicolumn{3}{|l|}{ Previous information } \\
\hline Yes & 6 & 30 \\
\hline No & 14 & 70 \\
\hline \multicolumn{3}{|l|}{ Source of information } \\
\hline Family members & 4 & 20 \\
\hline Health professionals & 2 & 10 \\
\hline \multicolumn{3}{|l|}{$\begin{array}{l}\text { History of gastrointestinal } \\
\text { disorders }\end{array}$} \\
\hline No & 20 & 100 \\
\hline
\end{tabular}

reported with a mild level of menopausal symptoms, and $20 \%$ of them had a moderate level of menopausal symptoms. It is also noticed that, in the post-test the severity of menopausal symptoms has come down with a mean of $15.6 \pm 2.633$.

- Table 3 shows the domain wise comparison of menopausal symptoms scores among women. It depicts that in the pretest, the domain on the psychological scale of the participants were with mean \pm SD of $11.10 \pm 0.738$, wherein the post-test the participants were with mean \pm SD of $7.30 \pm$ 2.293. During the pretest in Somato vegetative scale, the participants were with mean of \pm SD of $8.50 \pm 1.581$, whereas, in the post-test, with mean \pm SD of $6.40 \pm 1.776$ ). It shows that in pretest the domain on the urogenital scale, the participants were with mean \pm SD of $8.70 \pm 0.823$, whereas, in the post-test they were with mean \pm SD of $3.40 \pm 0.699$.

Data in - Table 4 show that the mean post-test menopausal symptom score was $15.60 \pm 2.633$ which was lower than the mean pretest score $28.420 \pm 1.476$ and the calculated 
Table 2 Classification of menopausal symptoms among participants (pre and post intervention)

\begin{tabular}{|l|l|l|l|l|l|}
\hline \multirow{2}{*}{$\begin{array}{l}\text { Level of menopausal } \\
\text { symptoms }\end{array}=20$} & \multirow{2}{*}{\begin{tabular}{l} 
Category \\
\cline { 3 - 6 }
\end{tabular}} & & \multicolumn{2}{|c|}{ Pespondents } & \multicolumn{3}{c|}{ Post-test } \\
\cline { 3 - 6 } & & $\begin{array}{l}\text { Frequency } \\
(f)\end{array}$ & $\begin{array}{l}\text { Percentage } \\
(\%)\end{array}$ & $\begin{array}{l}\text { Frequency } \\
(f)\end{array}$ & $\begin{array}{l}\text { Percentage } \\
(\%)\end{array}$ \\
\hline Mild & $0-14$ & - & - & 16 & 80 \\
\hline Moderate & $15-29$ & 14 & 70 & 4 & 20 \\
\hline Severe & $30-44$ & 6 & 30 & & $\mathbf{2 0}$ \\
\hline Total & & $\mathbf{2 0}$ & $\mathbf{1 0 0}$ & $\mathbf{1 0 0}$ \\
\hline
\end{tabular}

Table 3 Domain wise comparison of menopausal symptoms score among women (pre- and post-interventional)

\begin{tabular}{|l|l|l|l|l|}
\hline \multirow{2}{*}{$\begin{array}{l}\text { Domains } \\
n=20\end{array}$} & \multicolumn{2}{|c|}{ Pretest } & \multicolumn{2}{c|}{ Post-test } \\
\cline { 2 - 5 } & Mean & SD & Mean & SD \\
\hline Psychological scale & 11.10 & 0.738 & 7.30 & 2.293 \\
\hline Somato vegetative scale & 8.50 & 1.581 & 6.40 & 1.776 \\
\hline Urogenital scale & 8.70 & 0.823 & 3.40 & 0.699 \\
\hline
\end{tabular}

Abbreviation: SD, standard deviation.

Table 4 Effectiveness of a mind-body approach on menopausal symptoms using a paired $t$-test

\begin{tabular}{|l|l|l|l|l|l|}
\hline $\begin{array}{l}\text { Menopausal symptoms } \\
\boldsymbol{n}=\mathbf{2 0}\end{array}$ & Mean & SD & Mean difference & $t$-Value & $p$-Value \\
\cline { 1 - 4 } Pretest & 28.420 & 1.476 & 12.600 & 13.011 & $p<0.001$ \\
\cline { 1 - 3 } & 15.60 & 2.633 & & & \\
\hline
\end{tabular}

Abbreviation: SD, standard deviation.

$t$-value $t=10.157$ is greater than the table value $(p<0.001)$ at 0.001 level of significance. Hence, it is evident that the practice of mind-body approach was effective in reducing the intensity of menopausal symptoms among women.

\section{Discussion}

Psychological and behavioral interventions as well as yogic measures play a major role in reducing menopause-related symptoms. The present study shows the baseline information that, the majority (80\%) of the participants were in the age group of 51 to 53 years and had high school education (60\%). Similar findings were shown in a research study conducted by Enjezab et al in Iran who reported that the mean age of the women was $50.89 \pm 2.527$ years in the intervention group and $49.67 \pm 2.733$ years in the control group and majority of them had primary education level. ${ }^{13}$ Wong et al in China shows that the average age was $52.0 \pm 3.09$ years and $60.9 \%(n=120)$ participants were in the perimenopausal stage during the intervention and the present study shows that $50 \%$ of the participants attained menopause between 49 and 50 years. ${ }^{14}$ The present study reported a reduction of total MRS scores mainly on the psychological and Somato vegetative domains after the introduction of the mind-body approach. On a psychological scale, the participants had the maximum score of 10 with mean $11.10 \pm 0.738$, whereas, in the post-test, the score was 4 with a mean of $7.30 \pm 2.293$. In
Somato vegetative scale, the maximum score among women was 7 with a mean of $8.50 \pm 1.581$ whereas, in the post-test, the score was 4 with a mean of $6.40 \pm 1.776$. These findings are in far from the study findings by Wong et al in China which demonstrated that the effect of mindfulness-based stress reduction on the subscales of Greene Climacteric Scale revealed a significant reduction of anxiety $(F[1,35]=7.53, p$ $=0.007)$ and depression $(F[1,21]=4.78, p=0.031)$ but not on vasomotor, sexual, and urogenital subscales of menopausal symptoms. ${ }^{14}$

The study findings revealed that prior to intervention most of the subjects reported having moderate menopausal symptoms with $30 \%$ having severe menopausal symptoms. The intervention of the mind-body approach resulted in the mean post-test menopausal symptom score i.e., 15.60, which was lower than the mean pretest scores, 28.420. The calculated $t$-value $(t=13.011)$ is greater than the table value $(t \mathrm{tab}=1.83$, $p<0.001)$ at 0.001 level of significance and it revealed that the mind-body approach significantly helps the menopausal women in reducing the severity of menopausal symptoms. Similar findings reported in a study conducted by Joshi et al in Nagpur reported that the total MRS scores in both the study group and control group were high on day 1 ; mean total score of $9.37+7.28$ in the study group and $9+6.76$ in the control group, but on day 90 the mean score was significantly less $(P, 0.001)$ in the study group $(4.36+4.8)$ compared with the control group $(9.2+6.72) \cdot{ }^{15}$ The study findings consistent with another study 
conducted by Carmody reveals that participants daily HF severity score, decreased to $40 \%$ over 11 weeks of the assessment period. $^{16}$

\section{Implications of the Study}

Studies to date suggest that mind-body therapies may hold promise for reducing vasomotor and other symptoms of menopausal women. Empirical evidence gives a strong foundation to implement these practices as a routine in the care of menopausal women with various symptoms of menopause and also for reducing longer-term health effects of adverse metabolic and cardiovascular changes associated with menopause. This study has significantly contributed to the findings that mindbody therapies with the yoga-based practice were effective in minimizing the vasomotor symptoms, anxiety, and enhancing self-esteem among menopausal women.

\section{Conclusion}

The study concludes that mind-body approaches such as Mindfulness meditation had helped to reduce the frequency, severity, and bothersomeness of menopausal hot flashes. Besides, Mindfulness meditation also lessens the study participant's depressive symptoms, anxiety, and improved their self-esteem. The pilot study was found to be cost-effective and feasible to be made as a routine intervention for menopausal women to manage depressive symptoms as well to go ahead with the main study for the investigator. It is feasible to state that Mindfulness meditation may be applied as a complementary therapy toward post-menopausal women treatment.

\section{Authors' Contributions}

T.T. conceptualized the study, contributed to protocol development, data collection, analysis, and final writing of the report. N.K. and A.K. were guide and co-guide for the research project. F.D.S. was a member of the doctoral committee for the study. Under the guidance of N.K., A.K., and F.D.S. the study topic and research methodology were finalized and the research protocol was developed. The pilot study was supervised, and the data analysis and the draft of the article were corrected and finalized for publication.

\section{Conflict of Interest}

None declared.

\section{References}

1 World Health Technical Report Series. Research on the Menopause in the 1990's. Geneva: World Health Organization; 1996

2 Lewis V. Undertreatment of menopausal symptoms and novel options for comprehensive management. Curr Med Res Opin 2009;25(11):2689-2698

3 International Institute for Population Sciences, National Family Health Survey (NFHS-3), 2005-2006: India. Maharashtra: International Institute for Population Sciences; 2007

4 Utian WH. Psychosocial and socioeconomic burden of vasomotor symptoms in menopause: a comprehensive review. Health Qual Life Outcomes 2005;3:47

5 Lee A Kathryn and Moe E Karen Menopause. Principles and Practices of Sleep Medicine. 5th ed; 2011:1592

6 Woods NF, Mitchell ES. Symptoms during the perimenopause: prevalence, severity, trajectory, and significance in women's lives. Am J Med 2005;118(suppl 12B) :14-24

7 Statistics Korea, Population Projections for Korea: 2010-2060. Daejeon: Statistics Korea; 2011

8 Unni J. Third consensus meeting of Indian Menopause Society (2008): a summary. J Midlife Health 2010;1(1):43-47

9 Cooper GS, Sandler DP. Age at natural menopause and mortality. Ann Epidemiol 1998;8(4):229-235

10 Vaze N, Joshi S. Yoga and menopausal transition. J Midlife Health 2010;1(2):56-58

11 Johnson A, Roberts L, Elkins G. Complementary and alternative medicine for menopause. J Evid Based Integr Med 2019; 24:X19829380

12 World Medical Association. World Medical Association Declaration of Helsinki: ethical principles for medical research involving human subjects. JAMA 2013;310(20):2191-2194

13 Enjezab B, Zarehosseinabadi M, Farzinrad B, Dehghani A. The effect of mindfulness-based cognitive therapy on quality of life in perimenopausal women. Iran J Psychiatry Behav Sci 2019;13(1):e86525

14 Wong C, Yip BH, Gao T, et al. Mindfulness-based stress reduction (MBSR) or psychoeducation for the reduction of menopausal symptoms: a randomized, controlled clinical trial. Sci Rep 2018;8(1):6609

15 Joshi S, Khandwe R, Bapat D, Deshmukh U. Effect of yoga on menopausal symptoms. Menopause Int 2011;17(3):78-81

16 Carmody J, Crawford S, Churchill L. A pilot study of mindfulnessbased stress reduction for hot flashes. Menopause 2006;13(5): 760-769 